\title{
Mixed signal transportation for the EMBRACE antenna tiles
}

\author{
T. Berenz
}

\author{
Max-Planck-Institut fuer Radioastronomie (MPIfR), Auf dem Huegel 69, 53121 Bonn, Germany \\ e-mail: tberenz@mpifr-bonn.mpg.de
}

\begin{abstract}
This paper describes the transmission of the digital control signals, analogue RF signals and the power distribution of EMBRACE. It is shown how it is possible to transmit digital control data in full-duplex mode, analogue IF signals from $400 \mathrm{MHz}$ to $1600 \mathrm{MHz}$ and the supply current for the antenna tiles on the same coaxial cable. To keep the costs low, an completely symmetric design for the antenna tile and for the CDC unit is used. It consists of a active circulator, a diplexing structure, and a dedicated impedance transformer to match the impedance of the coaxial cable used.
\end{abstract}

\section{Introduction}

The EMBRACE demonstrator consists of several subparts. Here the signal transportation between the antenna tile and the CDC board will be explained.

The main goal was to transmit several signals on a single coaxial cable. The antenna tiles transmit an RF signal from $400 \mathrm{MHz}$ up to $1600 \mathrm{MHz}$ to the $\mathrm{CDC}$ units while the CDC units supply the antenna tile with a $48 \mathrm{~V} / 3$ A direct current via the same cable. For controlling every antenna tile, a digital control signal is needed. The decision was made to use the popular ethernet protocol, because it is widely used in computer networks, and it can be stated that this protocol will be used a long time in the future. The signal transportation part should be able to transmit these signals in full-duplex mode.

The antenna tiles are connected to the CDC boards via a $31 \mathrm{~m}$ coaxial cable for the Westerbork (Netherlands) station. A second station will be built in Nancay (France) with a cable length of about $28 \mathrm{~m}$ between the CDC boards and the tiles.

The technique used for EMBRACE is known as phased array or more precisely as dense aperture array. This means that, unlike the single-dish telescopes, a lot of identical components will be used, which leads to some additional design requirements. A summary of these is given below.

- The complete design has to be as cheap as possible. This means that the parts on the PCB should be able to be placed mechanically and a high level of integration on a PCB should save as many connectors as possible.

- The circuit should be designed such that tolerances in the parts used have no effect on the function. Hand-tuneable parts are not possible because of the huge quantity of boards needed.

- The 10 MBit ethernet signal should be transmitted in fullduplex mode with a good isolation from the analogue path.

- The design has to be able to transmit the supply current (48 $\mathrm{V} / 3 \mathrm{~A}$ DC) from the CDC unit to the antenna tiles.

- The RF path has to provide a bandwidth of $400 \mathrm{MHz}$ to $1600 \mathrm{MHz}$ with a return loss better than $-10 \mathrm{~dB}$ while the insertion loss should be as low as possible

All these requirements lead to the block diagram shown in figure 1 . As it can be seen, the design is completely symmetric,

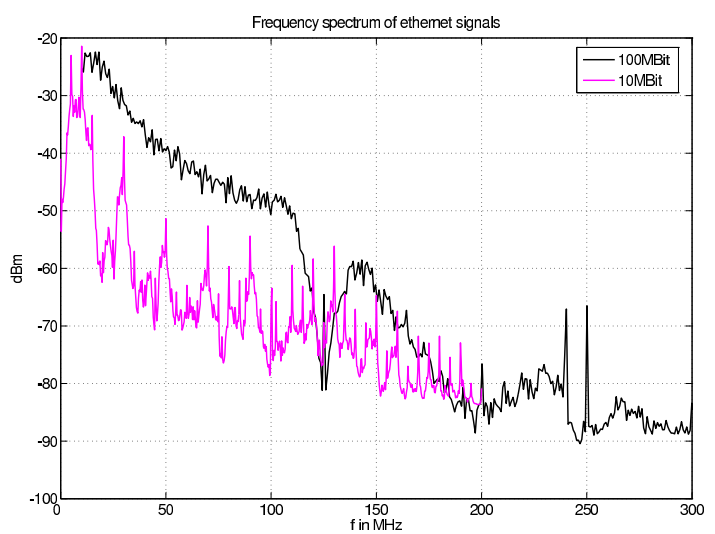

Fig. 2: Measured spectrum of the $10 \mathrm{MBit}$ and $100 \mathrm{MBit}$ ethernet signal

so that the same parts can be used for the antenna tile as well as for the CDC unit. The system mainly consists of a active circulator and a diplexer structure. Both will be described later in this paper.

\section{Active circulator}

To provide the full-duplex functionality while using only one coaxial cable, a circulator is needed to separate the transmitted and the received waves. To get an idea about the specifications needed for this circulator, one of the most important things to know is the frequency spectrum of the transmitted signal. This is shown for a 10 Mbit and a 100 Mbit ethernet signal in figure 2. For the $10 \mathrm{Mbit}$ ethernet a manchester coding is used. Therefore the frequency components are around $10 \mathrm{MHz}$, $30 \mathrm{MHz}, 50 \mathrm{MHz}$, and so on with most spectral power below $70 \mathrm{MHz}$

For the 100 MBit signal the situation is completely different. Here a 4 bit $/ 5$ bit scrambling mechanism is used before a three level coding. This leads to a spectral minimum around $125 \mathrm{MHz}\left(\frac{5 b i t}{4 b i t} 100 \frac{\mathrm{Mbit}}{\mathrm{s}}=125 \frac{\mathrm{Mbit}}{\mathrm{s}}\right)$ and a broad, powerful emis- 


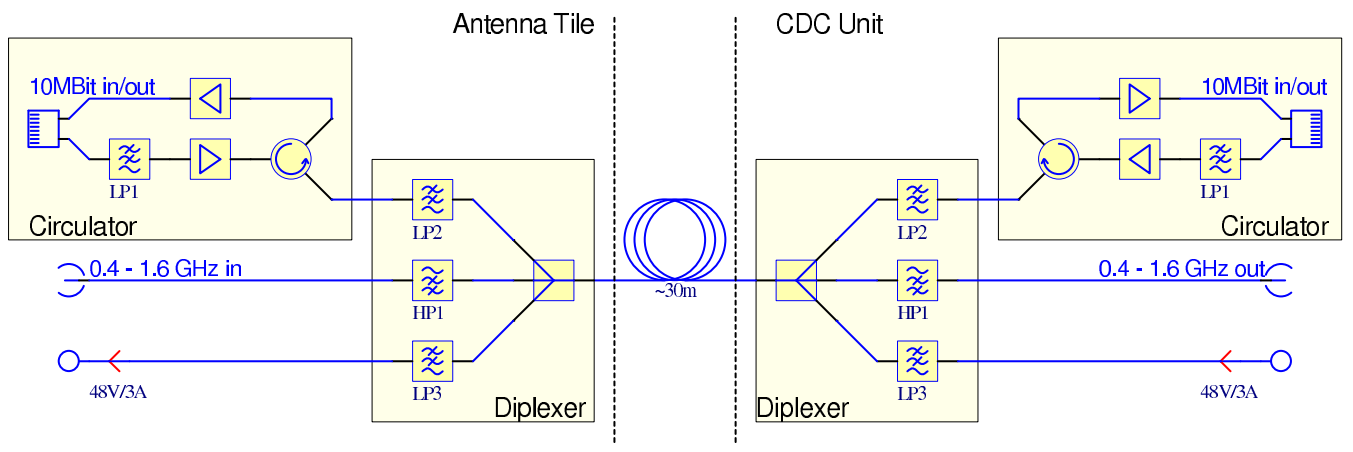

Fig. 1: Block diagram of the EMBRACE signal transportation part. The system consists of the antenna tile on the left side and the CDC unit on the right side connected with a standard $75 \Omega$ coaxial cable.

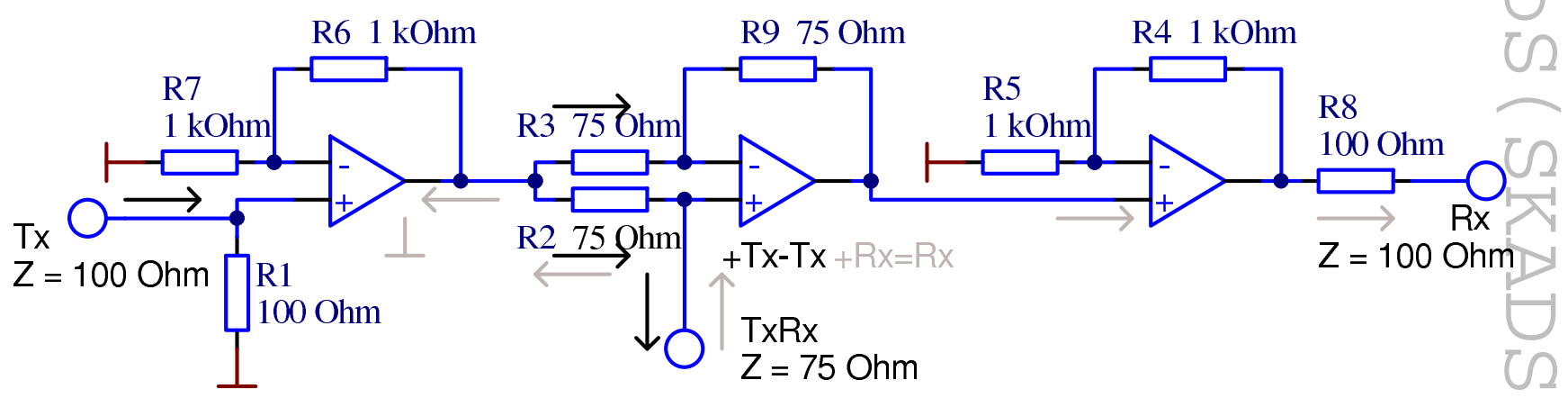

Fig. 4: Principle of operation of the active circulator

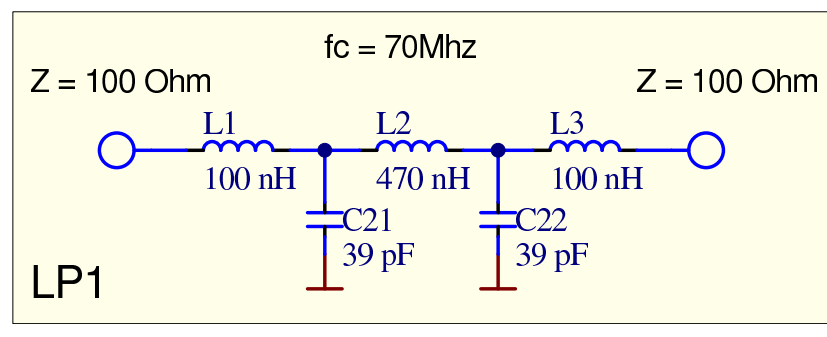

Fig. 3: Structure of the input lowpass filter of the active circulator

sion spectrum below these $125 \mathrm{MHz}$ as well as some strong harmonics at higher frequencies.

Both maximum frequencies are around $100 \mathrm{MHz}$ so it is possible to use an active circulator built up with fast operational amplifiers (OPA) to separate the waves on the coaxial cable.

To cut the frequency band of the incoming signal to a defined frequency, a lowpass filter is used in front of the active circulator. In figure 1 it is named as LP1. The design was chosen to provide a cut-off frequency of $70 \mathrm{MHz}$ with an input and output impedance of $100 \Omega$, which matches the impedance of the twisted pair ethernet cable. Figure 3 shows the structure of this lowpass filter. The principle of operation is shown in figure 4. The waves to transmit are marked with black arrows, while the receiving wave is marked with grey arrows. The structure consists of three amplifiers. The first (left) is used to amplify the incoming Tx signal and provide the signal sink for the incoming Rx signal. The second one (middle) is used to separate the two waves and the third one (right) is used to amplify the received signal. The connections to the circulator are a $100 \Omega$ impedance cable at the Tx port on left side of the schematic, a $100 \Omega$ impedance cable at the Rx port on the right side, and a $75 \Omega$ impedance cable at the TxRx port. The impedances of these cables are fixed, so the matching resistors R1, R2 and R8 also need to have these impedances.

When a signal to be transmitted is fed to the Tx port, the re sistor R1 will terminate the incoming wave with the cable's impedance. The signal then appears at the output of the first OPA amplified by $6 \mathrm{~dB}$. Since the middle OPA has the same structure for the inverting ( $\mathrm{R} 3=75 \Omega, \mathrm{R} 9=75 \Omega$ ), and the noninverting input $(\mathrm{R} 2=75 \Omega$, coaxcable impedance $=75 \Omega$ ) the signal will appear with the same level at both inputs and thus will be rejected.

The received signal is fed through the coaxial cable to the TxRx port. It is terminated with the $75 \Omega$ resistor $\mathrm{R} 2$ and the output of the first amplifier, which serves as a signal ground. Therefore the signal appears only at the noninverting input of the middle OPA. This OPA subtracts the signals on the inverting input (Tx) from the signals on the noninverting input $(\mathrm{Tx}+\mathrm{Rx})$, which means that only the receiving wave appears on the output of this OPA. A crucial point is the design of the PCB for this amplifier. Since it has to be very fast (several $100 \mathrm{MHz}$ ) the signal to transmit (black arrows) have to arrive at the same time at the inputs, which means that the line lengths from the output of the left amplifier to the input of the middle amplifier have to be exactly the same. To get good signal rejection at the output, the resistance ratios $\left(\frac{R 9}{R 3}\right)$ and $\left(\frac{R 2}{\text { coaxcable }}\right)$ have to be exactly the 


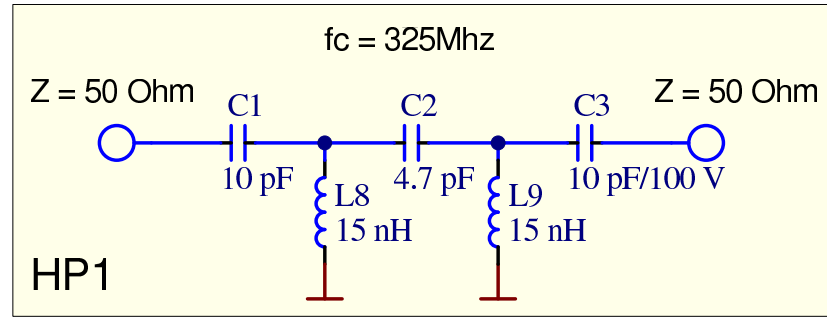

Fig. 5: Structure of the diplexer highpass filter

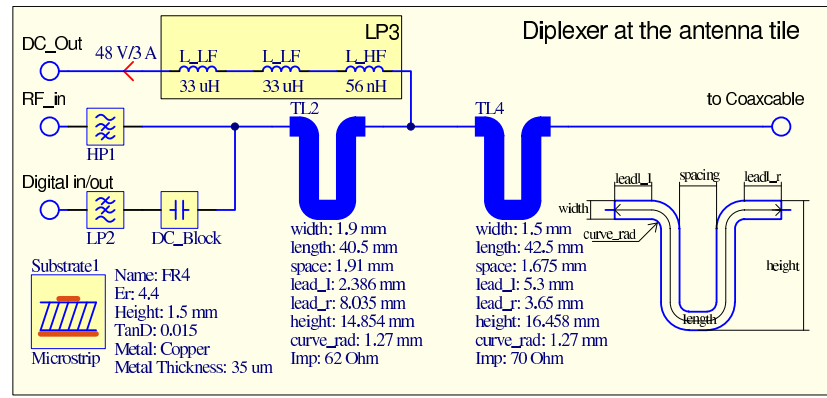

Fig. 6: Diplexing structure with the impedance transformer used

same. This requires also that the impedance at the TxRx port be constant across the complete frequency band, which means that LP2 of the block diagram (figure 1) needs to have a higher frequency than LP1.

The third operational amplifier is used to amplify the received signal by a factor of two. This compensates for the insertion loss of the coaxial cable.

\section{Diplexer}

As mentioned before, the complete circuit has to combine several signals on single coaxial cable. This is done by a diplexing structure which consists of several filters and an impedance transformer which will be explained here.

\subsection{RF path}

This path is used to transmit the RF signals from the antenna tile to the CDC unit. To separate it from the low-frequency control signals, a highpass filter with a cut-off frequency of $325 \mathrm{MHz}$ is used (see HP1 in figure 1). Its structure is shown in figure 5. The capacitor $\mathrm{C} 3$ has to withstand the supply voltage of $48 \mathrm{~V}$ so a $100 \mathrm{~V}$ type is used.

To match the filter impedance (50 $\Omega$ ) to the impedance of the coaxial cable (75 $\Omega$ ) two quarter wavelength transformers are used. The transformation is optimized as broadband as possible to get a good impedance match even at the band edges (400 MHz and $1600 \mathrm{MHz}$ ). The complete structure of this transformer is shown in figure 6.

For a frequency of $1 \mathrm{GHz}$ the right TL4 transforms the 75 $\Omega$ coaxial cable impedance to $65.42 \Omega$. The next line (TL2) then does another transformation to $59 \Omega$ (m1 in figure 7$)$. If the complete frequency range is taken into account, then the

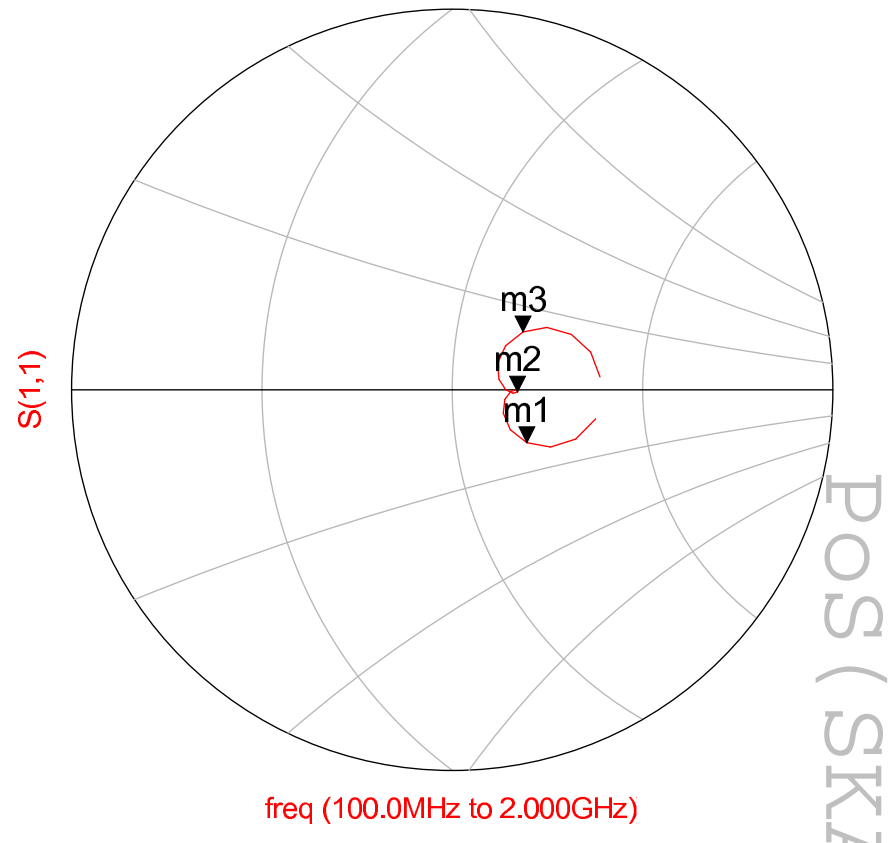

Fig. 7: Smith chart of the impedance transformation. The middle point represents the ideal matching point $(50 \Omega)$.

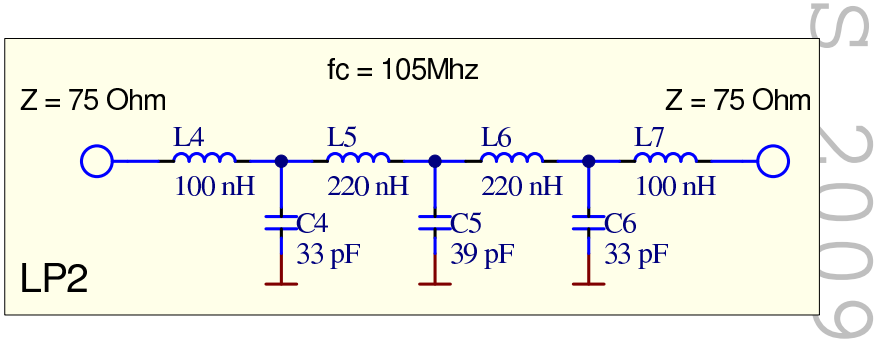

Fig. 8: Lowpass filter to separate the digital signals from the RF

impedance transformation ends at $60.2 \Omega$-j8.5 $\Omega$ for $400 \mathrm{MHz}$ (see $\mathrm{m} 1$ at figure 7 ) and at $59.5 \Omega+\mathrm{j} 9.2 \Omega$ for $1600 \mathrm{MHz}$ (see $\mathrm{m} 3$ in figure 7).

\subsection{LF path}

As mentioned before, the digital control signals are in the frequency band below $100 \mathrm{MHz}$. To keep these signals separate from the RF signals, a lowpass filter with a cut-off frequency of $105 \mathrm{MHz}$ is used. In figure 1 and figure 6 it is named as LP2 and the components used for this filter are shown in figure 8 .

\subsection{DC path}

A critical part of the diplexing structure is the DC biasing. The coils used there have to reject the complete frequency spectrum of the digital control signals (approx. $1 \mathrm{MHz}$ to $70 \mathrm{MHz}$ ) as well as the RF signals. After several iterations a combination of three coils led to a successful design. These are shown in the figure of the diplexing structure (figure 6). 


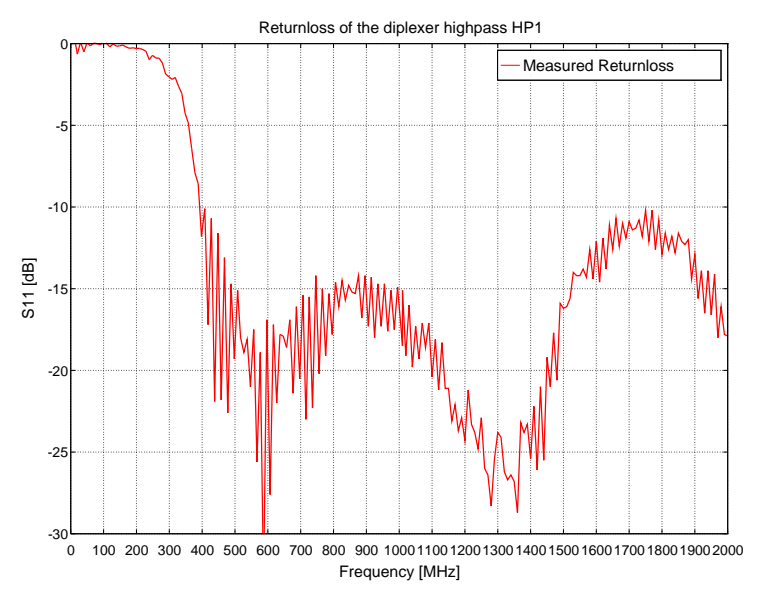

Fig. 9: Return loss of the RF path. The measurement was made for the complete link (diplexer - coaxial cable - diplexer)

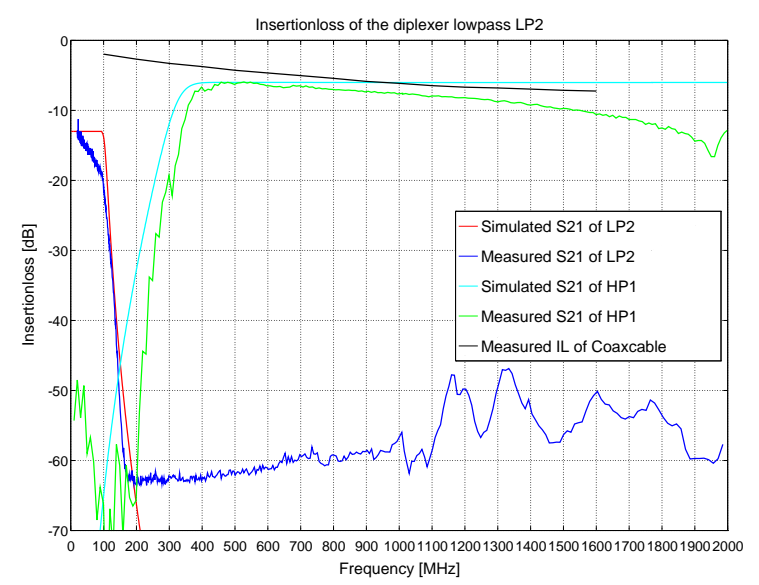

Fig. 10: Simulated and measured values for the diplexing filters (LP2 and HP1)

\section{Results}

The return loss of the RF path was specified to be better than -10dB from $400 \mathrm{MHz}$ up to $1600 \mathrm{MHz}$. Figure 9 shows the measurements. It can be seen that the specifications were met at the corner frequencies and were better than $-15 \mathrm{~dB}$ between $500 \mathrm{MHz}$ and $1.5 \mathrm{GHz}$.

The filter characteristics of the diplexing structure are displayed in figure 10. Both filters (LP2 and HP1) behave nearly as expected. The simulated values show a good matching to the measured ones. The deviation of the high pass filter slope from the simulated one can be explained by component tolerances and the parasitics caused by the board layout. The falling slope in the passband of the highpass filter is mainly caused by the attenuation of the coaxial cable, which is also plotted in the figure. The offset between the coaxialcable and the measured value of the highpass filter is about $1.5 \mathrm{~dB}$ and can be explained by the additional attenuation of the connectors used.

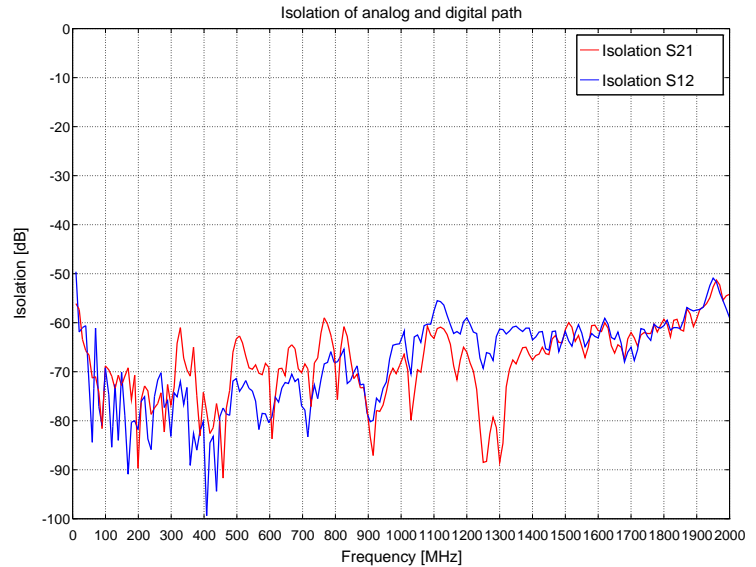

Fig. 11: Isolation from the analogue to the digital path of the diplexer

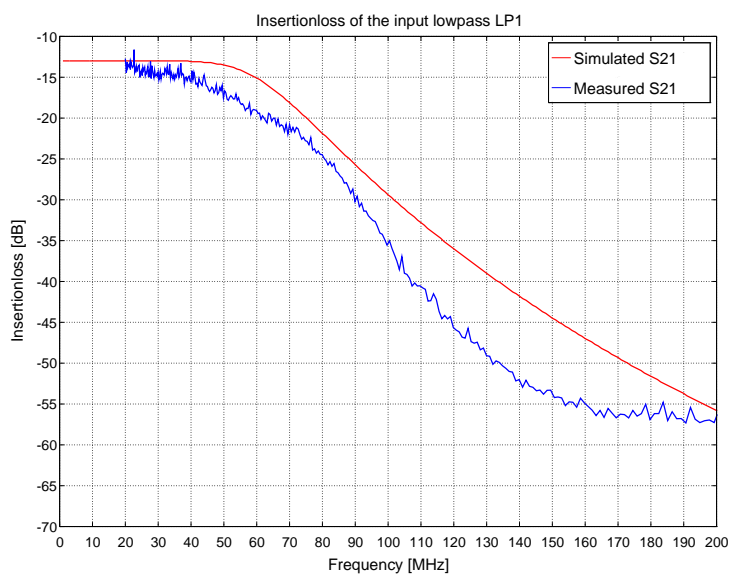

Fig. 12: Insertion loss of the input low-pass filter for the active circulator

The diplexer should separate the RF and digital signals from each other, therefore a good isolation between both paths is important. The achieved results are shown in figure 11. It is obvious that the isolation in most parts of the frequency band is better than $60 \mathrm{~dB}$.

As described before, LP1 is used to cut the frequency band of the incoming ethernet signal. The filter structure was designed with a cutoff frequency of about $70 \mathrm{MHz}$. Figure 12 shows the simulated and the measured filter slopes of this lowpass. The attenuation at frequencies above $100 \mathrm{MHz}$ is greater than $40 \mathrm{~dB}$. For the active circulator, a good isolation between the transmitting and receiving port is important. The measured values are shown in figure 13. It can be seen that the isolation is better than $15 \mathrm{~dB}$. Especially in the frequency band up to $20 \mathrm{MHz}$, where the most power of the ethernet spectrum is located, nearly 20 $\mathrm{dB}$ isolation from the $\mathrm{Tx}$ to the $\mathrm{Rx}$ port is reached. Now all measurements of the single filters were shown. The last measurement (see figure 14) shows the transmission of the digital link test pulse from the input of the first circulator, above the 


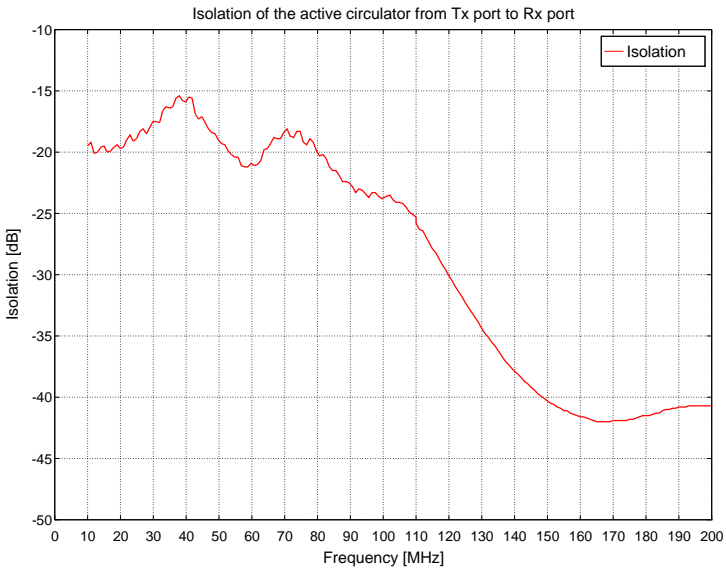

Fig. 13: Isolation from the Tx to the Rx port of the active circulator

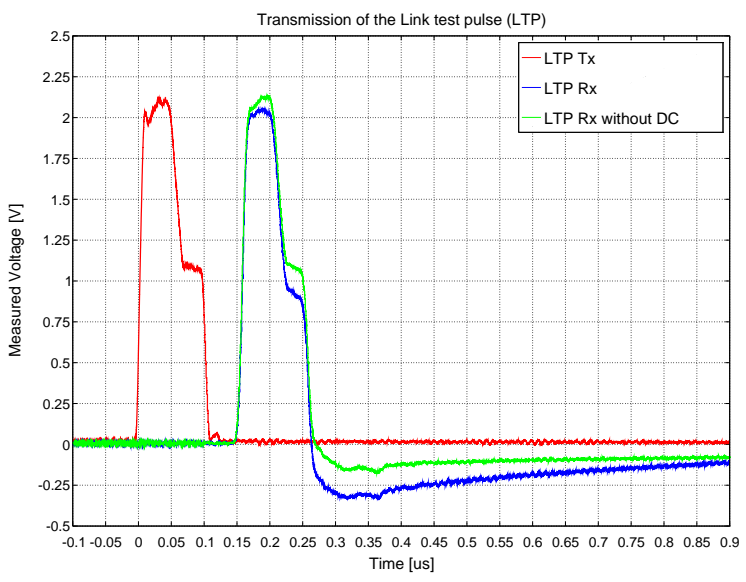

Fig. 14: Transmitted and received link test pulse (LTP)

diplexer, coaxial cable, second diplexer to the output of the second circulator, so across the whole system. The received link test pulse is delayed by $155.25 \mathrm{~ns}$. The difference between both received pulses arise from the DC feeding. If the DC feeding is connected to the RF path, the lowest frequency parts are not completely rejected, which means that they are terminated to ground. This leads to a slightly shift comparable with an AC coupling of the signal.

The quality of the signal transmission can be seen in the eye diagram in figure 15 . This diagram was taken during a $10 \mathrm{MBit}$ transmission of a large file from one PC to another. It is measured at the output of the circulator and displays $10^{6}$ samples. The eye is widely open in horizontal and vertical axes which means that the transmission quality is excellent.

\section{Conclusion}

The ethernet transmission behaves as expected. Although the filter slopes of the digital paths differ from the simulated values,

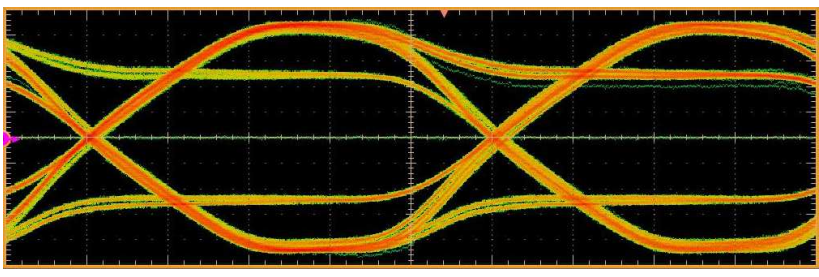

Fig. 15: Eye diagram of a $10 \mathrm{MBit}$ transmission

the $10 \mathrm{Mbit}$ transmission is realised and works properly. Every component used is commercially available, which helps to keep the cost low.

For the RF path, no tuning of the filter parameters is needed. The return loss is mainly better than $15 \mathrm{~dB}$. Only fixed values are used for the filters implemented so no component has to be tuned. This helps to keep the initial as well as the maintenance costs low.

The supply for the antenna tiles is also fed to the cable. 2 The circuit is able to supply the antenna tiles with $48 \mathrm{~V} / 3 \mathrm{~A}$. No influence can be observed on the RF signal and only little changes can be seen at digital LF signal.

In general, the complete structure behaves as planned. 